\title{
MOVILIZACIONES POR LA RED. LAS EVOLUCIONES DEL MOVIMIENTO OPEN
}

LA TRANSICIÓN A LO DIGITAL DE LA CULTURA Y EL CONOCIMIENTO: SUS EFECTOS EN LA CREACIÓN, LA INTERMEDIACIÓN Y LA PARTICIPACIÓN CULTURAL / DIGITAL TRANSITION IN CULTURAL AND KNOWLEDGE FIELDS: ITS EFFECTS ON ARTISTIC CREATION, INTERMEDIATION AND CULTURAL PARTICIPATION

\author{
Antonio Ariño Villarroya \\ Universidad de Valencia \\ ORCID iD: https://orcid.org/0000-0003-2683-4196 \\ antonio.arino@uv.es
}

Cómo citar este artículo/Citation: Ariño Villarroya, A. (2019). Movilizaciones por la red. Las evoluciones del movimiento open. Arbor, 195 (791): a489. https://doi.org/10.3989/ arbor.2019.791n1002

Recibido: 19 abril 2015. Aceptado: 8 marzo 2016.

RESUMEN: El movimiento open es el conjunto de luchas protagonizadas por individuos, foros, organizaciones e instituciones de muy diverso tipo que postulan una visión abierta, inclusiva y participativa de internet y de los bienes digitales. Consta de seis ramas fundamentales en función del campo sobre el que operan: el software, la comunicación científica, los materiales educativos, la creación cultural, la producción maker y la neutralidad de la red. Este artículo ofrece una visión sintética del movimiento, de sus características y aportaciones fundamentales.

PALABRAS CLAVE: Movimiento social; acceso abierto; neutralidad; sociedad digital.

\section{MOBILIZATIONS BY THE NET. THE EVOLUTIONS OF OPEN MOVEMENT}

Copyright: (c) 2019 CSIC. Este es un artículo de acceso abierto distribuido bajo los términos de la licencia de uso y distribución Creative Commons Reconocimiento 4.0 Internacional (CC BY 4.0).
ABSTRACT: Open Movement corresponds to the group of efforts of individuals, forums, organizations and institutions who or which postulate an open, inclusive and participative vision of both the Internet and digital goods. There are six main branches to the movement that relate to the different fields in which they operate: software, scientific communication, educational materials, cultural creation, maker production and the neutrality of the network. This paper offers a synthetic view of both this movement and its fundamental characteristics and contributions.

KEYWORDS: Social movement; open access; neutrality; digital society. 
"Nunca ha habido un momento como el actual, en el que una parte tan grande de nuestra cultura fuera posesión de alguien. Y sin embargo jamás ha habido un momento en el que la concentración de poder para controlar los usos de la cultura se haya aceptado con menos preguntas" (Lessig, 2005, p. 31).

“En el pasado reciente, la fracción, más que física esencialmente conceptual, del total de nuestra economía no ha dejado de crecer. Esta tendencia necesariamente ha desplazado el énfasis en la valoración de los bienes desde la propiedad física a la propiedad intelectual. Aunque el cambio puede parecer glacial, sus impactos sobre los riesgos legales y económicos comienzan a percibirse" (The Federal Reserve Board. Remarks by Chairman Alan Greenspan).

"Internet est notre société, notre société est Internet" (Blanc, 2011, 11 de marzo).

\section{INTRODUCCIÓN: LA CULTURA PARTICIPATIVA}

El 25 aniversario de la creación de la World Wide Web coincide con la primera reunión en Sao Paulo, en la primavera de 2014, de NETmundial, un foro global de "múltiples partes interesadas sobre el futuro de la gobernanza de internet", como ellos mismos se denominan (Global Multistakeholder Meeting on the Future of Internet Governance). Las ideas centrales de los documentos preparados para este encuentro tienen que ver con la apertura, la inclusividad y la participación en la red. Al mismo tiempo la Federal Communications Commission de Estados Unidos ha anunciado que va a revisar su política sobre la neutralidad de la red, generándose de inmediato un alud de protestas (Wheeler, 2014, 24 de abril).

Se ha llegado a este punto después de múltiples batallas en distintos frentes, que arrancan desde los años setenta y abarcan campos tan dispares como el acceso libre al software, al conocimiento científico, a las manifestaciones culturales, a los bienes comunes e incluso al hardware. Entre los pioneros, como Robert Stallman, Vint Cerf o Tim Berners-Lee, se difundió la visión de una internet abierta y libre. Desde entonces hasta las movilizaciones de 2012 contra los proyectos de ley SOPA (Stop Online Piracy Act) y PIPA (Protect Intellectual Property Act) en Estados Unidos y la AntiCounterfeiting Trade Agreement (ACTA) en Europa, se han producido muchas transformaciones, porque si algo caracteriza a la internet es su permanente evolución y sus cualitativos saltos adelante.

Para quienes todavía tuvieran una visión idílica o romántica de la red como el paraíso de la democratización cultural y política, los controles al acceso en Chi- na o en Arabia Saudí, las revelaciones de WikiLeaks y de Snowden sobre los procesos de vigilancia y control en Estados Unidos, han quebrado el espejo. La inspiración original de una Web -o un ciberespacio, como se ha dicho durante tiempo- abierta, libre, comunal y pública, se mantiene, pero hoy por hoy no es un dato de la realidad, sino un ideal que se antoja lejano.

¿Por qué son ahora tan importantes estas cuestiones legales sobre la propiedad intelectual y los derechos sociales de acceso a internet? En primer lugar, porque los recursos cognitivos constituyen el principal bien en disputa de la sociedad y economía actuales y porque el acceso libre a internet, en tanto que ecosistema global del conocimiento y de la información, forma parte de la visión de las libertades civiles en la era digital. Como sostiene Moulier, la digitalización de la economía y la sociedad nos ha introducido en un nuevo tipo de capitalismo -el cognitivo-, "una modalidad de acumulación en la que el objeto de acumulación se halla principalmente constituido por el conocimiento, que se convierte en la fuente principal del valor, así como en el lugar principal de la valorización" (Moulier Boutang, 2007, p. 94; Moulier Boutang, 2012, p. 175). En segundo lugar, porque se produce una transformación del modo de producción, donde hacer es innovar y compartir y donde la creatividad desborda el marco estrecho de la empresa. La producción de conocimientos nuevos no puede lograrse más que "a partir de la actividad cerebral colectiva movilizada en redes digitales interconectadas" (Moulier Boutang, 2007, p. 93). En tercer lugar, dado que la digitalización penetra todos los procesos sociales, el denominado tiempo libre también se convierte en un momento privilegiado para la innovación y la compartición. Ahora "se puede extraer mayor valor de la participación voluntaria de lo que se ha imaginado nunca, gracias a las mejoras en nuestra capacidad para conectarnos unos con otros y a las mejoras en nuestra imaginación de lo que es posible a partir de ella... La extraordinaria reducción del coste de la gobernanza pública, y el extraordinario crecimiento de la población conectada junta on line, significa que ahora podemos convertir agregaciones masivas de contribuciones pequeñas en cosas de valor perdurable" (Shirky, 2011, p. 161). Shirky denomina a este fenómeno "excedente cognitivo": consiste en tratar el tiempo libre de la gente como un agregado que permite producir nuevos conocimientos y sistemas de conocimiento; la creatividad en el tiempo libre se convierte en un activo que puede ser conectado a larga escala.

La digitalización ha cambiado, pues, tanto el sistema económico como el social y, sobre todo, se está trans- 
formando a sí misma. Nadie duda de que internet ha de ser regulado, la cuestión es por quién y cómo. El campo de fuerzas en juego es complejo porque los intereses de los actores son muy distintos. En este marco, el conjunto de luchas protagonizadas por individuos, foros, organizaciones e instituciones de muy diverso tipo que postulan una visión abierta, inclusiva, participativa, de internet y de los bienes digitales es lo que denominamos movimiento open (Ariño Villarroya, 2009).

La heterogeneidad de actores que lo componen, como veremos, es casi tan extensa como el espacio en el que actúan; en general se hallan especializados en distintos ámbitos, lo que puede contribuir a dar de ellos una imagen de fragmentación y de desarticulación en la medida en que pensamos con las claves de los movimientos en la calle; pero comparten una gramática discursiva que en cierto sentido se puede identificar con un cultura cívico-política. El núcleo gravitacional del campo semántico es el término open. La elección del calificativo no es arbitraria ni casual. Se halla presente en innumerables documentos y manifiestos, en los nombres de distintas corrientes y entidades y puede decirse que opera como principio ontológico (define cómo es la realidad), metodológico (cómo hay que producir) y estratégico (cómo hay que organizarse y luchar) (COAPI Principles).

Open Source, Open Access y Open Science son denominaciones consagradas para referirse a las ramas que defienden el acceso libre al software y al conocimiento científico (Subirats i Coll. El movimiento Open Access: Concepto e historia). Pero esta concepción se ha extendido progresivamente a otros ámbitos: a la docencia (con los Open Course Ware, Open Educational Resources y recientemente con los Massive Open On-line Courses, MOOC); se ha desparramado por las prácticas culturales artísticas y las formas de sociabilidad de la Web 2.0 o de las culturas fan; y finalmente, en los últimos cinco años, con la invención de las impresoras digitales y otras formas de producción física a partir de la digitalización, ha recalado en el movimiento Do It Yourself (DIY) y los makers, que crean repositorios de Open Hardware. Por tanto, el movimiento postula unas determinadas condiciones en la producción y transmisión simbólica de la sociedad red y desafía las vías de apropiación comercial privada; rechaza la reducción del conocimiento a mercancía o bien privado y reclama la construcción de un dominio público nuevo para el desarrollo de una cultura libre mediante la accesibilidad abierta (incluyente y no restringida por códigos propietarios).

Este artículo ofrece una visión sintética del movimiento y sus ramificaciones, de sus características y aportaciones fundamentales y esboza una aproximación a sus bases sociales y formas de organización. Se enmarca en el proyecto NOMS que estudia los nuevos objetos mundo-sociales que surgen en la era digital, siendo uno de ellos no ya la aparición de movimientos que utilizan las herramientas digitales, sino que versan sobre la naturaleza de la sociedad digital y de internet como su ecosistema cultural, al tiempo que abordan la cuestión de las libertades civiles y la redefinición de la esfera pública.

\section{LAS RAMAS DEL MOVIMIENTO OPEN}

Los movimientos sociales no solo están utilizando las redes sociales como un medio para la transmisión de información y para la comunicación, sino también para coordinar su acción. Además, la mayoría de estos movimientos y revueltas también se ocupan, aunque sea tangencialmente, de la naturaleza de la red y de los problemas crecientes que se plantean con ella y en ella: de acceso, propiedad, seguridad y privacidad.

Estos aspectos -los derechos de acceso a internet, la naturaleza abierta y libre de la red y su conversión en un medio de coordinación para la acción política participativa y crítica- constituyen el objeto de un movimiento socio-digital menos visible, menos cargado del dramatismo y fulgor (y del romanticismo) de las protestas y revueltas en la calle, sea en la plaza Tahrir, en la Puerta del Sol o en la plaza Maidán. Y dado que en la economía digital aparecen nuevos bienes, como el software, el hardware, el wetware, el netware y el shareware, las movilizaciones se producen también en dichos frentes y dan lugar a distintas ramas.

En este artículo sostenemos que existen seis ramas fundamentales, que han ido surgiendo de la propia evolución de la internet, y que con frecuencia se califican a sí mismas cada una de ellas como movimientos. En primer lugar, la movilización nació con la defensa del software libre; muy pronto se produjo la lucha a favor del acceso abierto a los resultados de la investigación científica; un poco más tarde aparecieron los grupos defensores de la creación de contenidos académicos abiertos para la docencia. Durante todo este tiempo, en paralelo, se extendió la concepción de la cultura, el arte y los productos para el ocio como bienes comunes; con la invención de las impresoras digitales y la aparición de la producción personalizada, irrumpen las propuestas del hardware libre entre los autodenominados makers; y finalmente, se ha agudizado el problema sobre la gobernanza de internet y sus consecuencias para la democracia y la esfera pública global. 
Tabla 1. Evolución de hitos significativos para el movimiento open

\begin{tabular}{|c|c|c|c|c|c|c|c|c|c|c|c|c|}
\hline 1982 & 1983 & 1991 & \begin{tabular}{|l|}
$1991-$ \\
1993 \\
\end{tabular} & 1997 & 1999 & 2001 & 2001 & 2001 & 2001 & \begin{tabular}{|l|}
$2001-$ \\
2004 \\
\end{tabular} & \begin{tabular}{|l|}
$2002-$ \\
2003 \\
\end{tabular} & 2004 \\
\hline Vint Cerf & \begin{tabular}{|l|} 
Richard \\
Stallman
\end{tabular} & & $\begin{array}{l}\text { Tim } \\
\text { Berners } \\
\text { Lee }\end{array}$ & $\begin{array}{l}\text { Marc } \\
\text { Adrees- } \\
\text { sen }\end{array}$ & \begin{tabular}{|l} 
Sean \\
Parker \\
Shwam \\
Fanning \\
\end{tabular} & $\begin{array}{l}\text { Lawrence } \\
\text { Lessig }\end{array}$ & MIT & $\begin{array}{l}\text { Lawrenc } \\
\text { Lessig }\end{array}$ & $\begin{array}{l}\text { Jimmy } \\
\text { Wales }\end{array}$ & Suecia & \begin{tabular}{|l} 
Bethesda \\
Budapest \\
Berlin
\end{tabular} & $\begin{array}{l}\text { Tim } \\
\text { O'Really }\end{array}$ \\
\hline \begin{tabular}{|l} 
Protoco- \\
los TCP/ \\
IP \\
\end{tabular} & $\begin{array}{l}\text { Open } \\
\text { Source }\end{array}$ & $\begin{array}{l}\text { Internet } \\
\text { Society }\end{array}$ & WWW & Netscape & $\begin{array}{l}\text { Napster } \\
\text { P2P }\end{array}$ & \begin{tabular}{|l|} 
Creative \\
Commons
\end{tabular} & $\begin{array}{l}\text { Open } \\
\text { Course } \\
\text { Ware }\end{array}$ & \begin{tabular}{|l|} 
Creative \\
Commons
\end{tabular} & Wikipedia & \begin{tabular}{|l} 
The \\
Pirate \\
bay \\
\end{tabular} & $\begin{array}{l}\text { Open } \\
\text { Access }\end{array}$ & Web 2.0 \\
\hline 2006 & 2006 & 2006 & 2007 & \begin{tabular}{|l|}
$2007-$ \\
2008
\end{tabular} & 2008 & $\begin{array}{l}2008- \\
2012\end{array}$ & 2012 & 2013 & 2014 & 2014 & 2014 & \\
\hline $\begin{array}{l}\text { Hacker- } \\
\text { spaces }\end{array}$ & \begin{tabular}{|l|} 
Rickard \\
Falkvinge
\end{tabular} & \begin{tabular}{|l|} 
Thorsten \\
Wirth
\end{tabular} & & $\begin{array}{l}\text { Julian } \\
\text { Assange }\end{array}$ & & $\begin{array}{l}\text { MIT } \\
\text { Harvard } \\
\text { Coursera }\end{array}$ & $\begin{array}{l}\text { Acciones } \\
\text { contra } \\
\text { SOPA, } \\
\text { PIPA, } \\
\text { ACT } \\
\end{array}$ & $\begin{array}{l}\text { Unión } \\
\text { Europea }\end{array}$ & $\begin{array}{l}\text { Reunión } \\
\text { en Brasil }\end{array}$ & \begin{tabular}{|l} 
Regular \\
la neu- \\
tralidad \\
de la \\
red \\
\end{tabular} & $\begin{array}{l}\text { Defensa de } \\
\text { la neutra- } \\
\text { lidad de la } \\
\text { red }\end{array}$ & \\
\hline Makers & $\begin{array}{l}\text { Partido } \\
\text { Pirata } \\
\text { Suecia } \\
\end{array}$ & \begin{tabular}{|l|} 
Partido \\
Pirata \\
Alemania \\
\end{tabular} & $\begin{array}{l}\text { Open Net } \\
\text { Initiative }\end{array}$ & $\begin{array}{l}\text { Wiki- } \\
\text { Leaks }\end{array}$ & \begin{tabular}{|l|} 
Global \\
Network \\
Initiative \\
\end{tabular} & MOOC & \begin{tabular}{|l} 
Amplia \\
moviliza- \\
ción
\end{tabular} & \begin{tabular}{|l|} 
Protocolo \\
Open \\
Access \\
\end{tabular} & $\begin{array}{l}\text { Netmun- } \\
\text { dial }\end{array}$ & FCC & $\begin{array}{l}\text { Grandes } \\
\text { compañías } \\
\text { de internet }\end{array}$ & \\
\hline
\end{tabular}

Fuente: elaboración propia.

Todas estas ramas provienen y se alimentan, como hemos comentado, de un tronco cultural común, que se observa al registrar la gramática utilizada y que puede ser analizado distinguiendo cuatro marcos fundamentales de referencia: open (apertura), free (libertad-gratuidad), commons (comunalidad) y public (carácter público de los bienes que se hallan en juego) ${ }^{1}$.

En los apartados siguientes presentaremos las características de las distintas ramas y su entronque con la defensa de una cultura libre y una reconstrucción de la esfera pública en condiciones de comunicación móvil.

\section{La producción cooperativa de software}

Los programadores informáticos fueron los primeros en introducir pautas de experimentación mediante la lógica y la cultura open. Utilizaron la expresión Open Source para referirse a las condiciones abiertas en que debía facilitarse el acceso a las instrucciones o códigos de los equipos informáticos, pero también para designar la forma de trabajar de las comunidades desarrolladoras de programas y aplicaciones.

Cuando a finales de los años setenta y principios de los ochenta del pasado siglo las empresas se propusieron hacer negocio con la distribución de los códigos fuente, Richard Stallman, profesor del MIT, creó una fundación para la defensa del libre acceso (1983), pero esta visión solo alcanzaría éxito a partir de 1991, cuando Linus Torvalds convocó a voluntarios de todo el mundo a trabajar en el proyecto Linux. La aparición de la World Wide Web y el incremento mundial del número de programadores crearon las condiciones para la existencia de una masa crítica de colaboradores, basada en la participación voluntaria, asentada en una motivación interna, para la producción de software abierto. De esta forma surgieron "comunidades virtuales de desarrolladores y usuarios" entre las que cabe destacar Linux, Apache, Mozilla y Open Office.

Como han defendido diversos expertos (Raymond, 2001; Shirky, 2008), con Linux se produjo un cambio sustantivo en las reglas de juego de la organización del trabajo, convirtiéndose en el primer proyecto que hizo un esfuerzo consciente y exitoso para adoptar el mundo entero como su base de producción de talento. Raymond ha señalado que, si bien crear código sigue siendo una actividad habitualmente solitaria, las contribuciones o hacks se logran mediante el ensamblaje de las capacidades mentales de numerosas personas. De esta manera, dichas comunidades, ligadas por la conectividad de la red, aportan un nuevo método de producción que se asemeja más a la dinámica de un bazar que a la construcción orquestada de una catedral.

Estas redes de cooperación -difusas, contingentes y flexibles- incorporan las buenas ideas vengan de donde vengan, sin costes de transacción y de gerencia, porque no hay empleados sino contribuyentes; y se basan, no en una concepción de la propiedad corporativa, sino de la inteligencia cooperativa. Se hallan abiertas al fracaso, porque su objetivo es conseguir aportaciones y elegir las que contribuyen a la mejora y la calidad del producto.

Además de un método de trabajo eficaz, basado en la reducción de costes de transacción y en la poten- 
ciación de las formas de coordinación, propiciados por la Web, una parte importante de este movimiento defiende también una filosofía moral: el software no puede ser tratado como una mercadería, sino como una utilidad o servicio público que capacita para el desarrollo de recursos en común. El movimiento defiende el derecho a compartir información y el derecho a ser, no solo consumidores de conocimiento y cultura, sino también creadores (Moglen, 2003, 29 de junio). Esta lógica, fundada en la agregación de pequeñas contribuciones individuales para un proyecto abierto, colectivo y público, se ha ido extendiendo con posterioridad a múltiples campos.

\section{La producción abierta del saber (Open Access)}

La visión de apertura y libertad se ha extendido al ámbito de la investigación y del conocimiento científico: se conoce como Open Access y se entiende por tal el acceso online libre, inmediato, permanente, completo, a material digital científico y académico, fundamentalmente a artículos de investigación publicados en revistas basadas en la revisión por pares. Esta concepción se ha ido decantando en declaraciones como las de Budapest (2002), Bethesda (2003) y Berlín (2003), dejando claro que cualquier usuario individual, en cualquier lugar, debe poder enlazar, leer, bajar, almacenar, imprimir, utilizar y extractar el contenido digital del correspondiente documento (Suber, 2012).

Un concepto íntegro de Open Access no solo propone facilitar el acceso a la lectura de textos y a bases de datos, sino también al uso y a la transformación creativa y supone eliminar dos restricciones o barreras fundamentales: la de los precios (gratuidad de acceso) y la de las licencias (derechos de autor y de reproducción), que se refiere a la libertad de uso (Suber, Open Access Overview. Focusing on open Access to peer-reviewed research articles and their preprints).

El Open Access no es incompatible con el copyright, con la revisión por pares y ni siquiera con la obtención de beneficios, puesto que su rasgo más definitorio se encuentra en la eliminación de las barreras de acceso al conocimiento. Sin embargo, al menos quienes postulan un Libre Knowledge y los firmantes de la Declaración de Washington sobre la Propiedad Intelectual y el Interés Público (2011), consideran imprescindible la puesta en cuestión del actual modelo de copyright ${ }^{2}$, para que el conocimiento pueda ser adquirido, interpretado y aplicado libremente, reformulado de acuerdo con las propias necesidades y compartido con otros.
Existe una contradicción inaceptable entre, de un lado, la creciente extensión de los derechos y de las patentes que restringen y retardan la difusión del conocimiento y, de otro, las oportunidades generadas por las nuevas tecnologías. La comunidad científica siempre ha tratado de facilitar un acceso rápido a los resultados de investigación y hoy no solamente es posible reducir drásticamente el tiempo entre el hallazgo y su publicación sino también incrementar la escala de difusión. Por otro lado, estas tecnologías potencian nuevas formas de interacción, coordinación y organización entre científicos y crean nuevos objetos de conocimiento, como sucede en el ámbito de las ciencias de la vida, en la física o en la química, con la utilización de la computación distribuida (GRID).

Como recomienda Science Commons (Barcelona, 2008), para apoyar este nuevo modelo de investigación y de innovación -interdependiente, internacional e intensivo en el uso de datos y series- es preciso actuar en varios frentes: acceso abierto a las publicaciones resultantes de la investigación financiada; incorporación al dominio público de los datos de investigación (incluidos metadatos y protocolos) y creación de una ciber-infraestructura abierta. Entre los instrumentos más importantes, de carácter legal, económico, técnico, etc., que se han desarrollado destacan las licencias abiertas (GNU, Copyleft, Creative Commons), las publicaciones (Open Journals), los repositorios digitales ${ }^{3}$ y los estándares y protocolos para las infraestructuras de datos primarios. En 2004, el directorio de Journals de acceso abierto registraba 1.114 revistas, mientras que en 2014 pasaron a ser casi 10.000 (Directory of Open Access Journals).

Pero no solamente hay razones de política científica para defender el Open Access, sino también de política social y económica: dado que la mayoría de la financiación de la investigación procede de fondos públicos y se realiza por profesionales que trabajan en organizaciones públicas, la sociedad que las financia tiene derecho a conocer con la mayor transparencia e inmediatez los descubrimientos que pueden afectar a sus condiciones de vida. Esta es por ejemplo la posición de quienes firmaron en 2006 el HealthTrain. The Open Care Manifesto o de quienes han creado MedLine y, cada vez, de un mayor número de redes, instituciones y gobiernos. Así, la Unión Europea analizó el impacto del acceso abierto en un documento dado a la luz en agosto de 2013 y ha publicado con posterioridad su política sobre el asunto en el Horizonte 2020 (Nicol, Caruso y Archambault, 2013). 


\section{Contenidos educativos libres (Open Educational Re-} sources)

En 1998, David Wiley introdujo el concepto open content para expresar, por analogía, la idea de que los principios del open software también se podían aplicar a los contenidos culturales y educativos y creó la primera licencia abierta para ellos. Con el paso de los años, la expresión Open Educational Resources (OER) ha tenido un éxito mayor y sirve hoy para identificar la tercera rama del movimiento.

Mediante el apoyo de diversas fundaciones altruistas, instituciones y organizaciones internacionales (como UNESCO y OCDE) y la implicación de universidades y centros educativos de todo el mundo, se ha creado un movimiento global, basado en una pluralidad de iniciativas, que defiende la importancia de compartir el conocimiento de una manera abierta y libre para facilitar el aprendizaje de todas las personas en la era de las nuevas tecnologías. Una de las iniciativas pioneras en este ámbito y de mayor irradiación ha sido el Open Course Ware del MIT (OCW), un proyecto editorial electrónico para poner en la red, en acceso abierto, los materiales docentes correspondientes a cursos completos de las universidades. Este proyecto se ha visto desbordado recientemente por el nacimiento de los MOOC, cursos masivos online en abierto que se expanden por numerosas universidades.

Los OER son "recursos de docencia, investigación y aprendizaje alojados en el dominio público o que gozan de un tipo de licencia de propiedad intelectual que permite su uso libre y su reutilización para otros fines por otras personas... incluyen cursos completos, módulos, libros de texto, descargas de video, textos, software y cualesquiera otros materiales, herramientas o técnicas utilizadas como soporte para el acceso al conocimiento" (Atkins, Brown y Hammond, 2007). El movimiento persigue la creación de una comunidad global online de aprendizaje, basada en la lógica de compartir contenidos y en la potenciación de las redes sociales.

\section{Licencias abiertas para una cultura libre}

El movimiento open desborda los ámbitos técnico, científico y académico, y se ocupa también de la creación, producción y distribución de cultura en general, de la posibilidad de mezclar y reutilizar y de la capacidad creativa de la gente corriente en la vida cotidiana (Jenkins, 2006); incluye tanto la producción en la esfera del arte (literario, musical, etc.) como en las expresiones rutinarias del tiempo de ocio; se plasma en la práctica de descargas musicales o cinematográficas, así como en la producción, derivación y recreación de todo tipo de productos; en las prácticas de transformación de obras consagradas típicas de las culturas fan y en las batallas libradas por hackers y anonymous. La propia definición de conocimiento conlleva para el movimiento esta visión englobante: "cualquier tipo de contenido, información o datos, desde genes a geodatos, desde sonetos a estadísticas... que se puede usar, re-usar y redistribuir libremente por cualquier persona, sin restricciones legales, sociales o tecnológicas" (Hess y Ostrom, 2006).

La digitalización -se afirma- permite un mejor desarrollo de la propia naturaleza de la cultura y de las ideas puesto que, al subrayar su dimensión inmaterial, al desacoplar los significados de sus soportes materiales y presentarlos mediante series binarias, es posible realizar un número infinito de copias y distribuirlas a un coste mínimo.

De acuerdo con Moglen (2003, 29 de junio), en el siglo XXI se pueden distinguir dos clases de bienes que tienen un coste marginal cero. La primera es la configurada por los bienes funcionales (orientados a la utilidad) como el software computacional, los mapas o la información del genoma; en este caso, la producción sin relaciones de propiedad, compartiendo toda la información, genera bienes mejores; la segunda corresponde a bienes no funcionales, como los artísticoculturales y en estos la distribución "anarquista", sin exclusión de nadie, produce una diseminación mejor, más eficaz, que la mercantil.

Hay dos conflictos fundamentales en juego: uno tiene que ver con la distribución de los bienes simbólicos; el otro, con la autoría y la propiedad intelectual. El primero requiere un cambio de modelo de negocio; el segundo de regulación jurídica.

La distribución de los bienes simbólicos digitalizados (música, cine, noticias, ficción escrita, etc.) se ha planteado por parte de la industria editora mediante el modelo económico gutembergiano, al mismo tiempo que los consumidores descubrían que la difusión digital ilimitada permitía recoger los bienes culturales en los servidores de descargas como si fuesen bayas del bosque en una economía de recolección. Ahora bien, el principal problema para una cultura libre no radica en la mal denominada "piratería", sino en la asfixia de la libertad por la omnipresencia de las patentes y de los restrictivos derechos empresariales. La recreación de un dominio comunal o público es imprescindible para el desarrollo de la creatividad, la innovación y la cultura libre. Esta posición parece re- 
frendada por cierta investigación empírica. Así, según (Camaerts y Meng, 2011), la compartición de música online y la infracción de los derechos de propiedad sostienen que el balance es ambivalente porque aparecen nuevas formas de economía y desde luego no se sostienen empíricamente las pretensiones de la gran industria musical.

Por otra parte, la defensa de una cultura libre, al menos por parte de Lawrence Lessig (autor de referencia e inventor de las licencias Creative Commons) no debe entenderse como una apología de la "barra libre" cultural o del acceso gratuito sino como una extensión de las libertades de la modernidad (libre albedrío e incluso mercado libre) y una limitación significativa de la santificación de la propiedad intelectual. De este modo, una cultura libre apoya y protege a los creadores e innovadores y lo hace de dos maneras: a) directa: concediendo derechos de propiedad intelectual; b) indirecta: limitando el alcance de dichos derechos, para garantizar que los creadores e innovadores que vengan después no se encuentren apresados por las protecciones del pasado.

Aún así, en cuanto a la propiedad intelectual queda un aspecto importante que hay que considerar: tanto en las creaciones amateurs como en las profesionales, cada vez más, el producto final es resultado de una cooperación y conversación extensas. Wikipedia invita a cada persona a participar como autor y editor y cada voz es obra de numerosas personas con contribuciones desiguales; en el universo del hipertexto los resultados que se obtienen ante una pantalla tras una búsqueda proceden de la cooperación de cientos y puede que de miles de colaboradores e incluso de algoritmos y robots; y las comunidades de digital folk o de práctica fan, estudiadas por Henry Jenkins, se basan en una visión de la propiedad intelectual como shareware (Jenkins, 2006).

\section{El retorno a los átomos (Open Source Hardware)}

Se conoce como movimiento Do It Yourself (DIY), a aquellas redes y personas que realizan trabajos domésticos o de aficionado por y para sí mismas. Este movimiento -leído en clave política- puede oscilar desde quienes militan en el mismo por una oposición explícita a la sociedad de consumo, su falta de sostenibilidad e impersonalismo (lema punk: "no importa si eres bueno, hazlo") hasta quienes sencillamente encuentran en el bricolaje una forma creativa de entretenimiento y de ahorro doméstico. Estos amateurs y hackers se diferencian de los profesionales, desde luego, por el modo de adquisición de las habilidades y seguramente también por su experticia o competencia, pero "siempre se distinguen por las motivaciones" (Shirky, 2011, p. 82). La esencia del amateurismo es una motivación intrínseca: hacer algo por el placer de hacerlo, por el amor que se le tiene.

Las nuevas tecnologías de la Web 2.0 suprimen otra diferencia entre ellos: los amateurs actuaban en espacios privados y domésticos, mientras que los profesionales lo hacían en el mercado o en el espacio público. Sin embargo, internet ha potenciado extraordinariamente la capacidad de captación de información (y de aprendizaje), de comunicación e interacción y de organización de los amateurs; pueden producir (make), crear, organizarse, compartir y hacerlo a gran escala, con fines comunitarios, cívicos o políticos; pequeñas contribuciones se pueden agregar y conectar ahora de forma masiva creando un valor duradero (Shirky, 2011, p. 161).

Al fenómeno de compartir a gran escala, se ha sumado otra innovación tecnológica, que Chris Anderson califica de giro copernicano en la revolución digital: los últimos diez años han gravitado en torno al descubrimiento de nuevas formas para crear, innovar y trabajar juntos en la Web; los próximos diez años se dedicarán "a aplicar todo ello al mundo real" generando la tercera revolución industrial (Anderson, 2012, p. 17). Esto es lo que ha comenzado a suceder con las máquinas que convierten los bits en átomos. De momento, se trata de máquinas rudimentarias de escaneo en 3D, impresoras de escritorio 3D, fresadoras, máquinas de coser, productoras de circuitos, etc. que pueden utilizar un número limitado de materiales como plástico, cerámica o madera.

En esta tercera revolución industrial la cuestión central no es cómo se hacen las cosas, sino quién las hace. Como señaló Neil Gershenfeld -director del Center for Bits and Atoms- las nuevas máquinas, al igual que sucedió en su momento con el ordenador personal, serán accesibles a toda la gente, a cualquiera. "Lo que será personalizado -dice- será nuestro mundo físico de átomos más que el mundo digital de los bits" (Gershenfeld, 2012, p. 49). Ha llegado la hora de la fabricación digital, que consiste en la capacidad para convertir datos en cosas y cosas en datos y esta conlleva la propiedad personal de los medios de producción (Gershenfeld, 2012, p. 49). “Esta revolución viene marcada por la participación en el proceso de producción de los propios usuarios, a través de la customización en masa, la producción cooperativa y la fabricación personal, simplificando y abaratando los sistemas de diseño 3D" (Fernández, 2012). 
La variación que ofrece la digitalización y la producción industrial personal mediante las máquinas digitales de producción doméstica, unida a la fuerza aportada por el amateurismo, se aúnan en el movimiento maker, que postula no solamente el free software, sino el Openhardware (como, por ejemplo, el proyecto Arduino). Al "usar y tirar" de la producción en serie, oponen la producción personal cooperativa; a la unidimensionalidad del consumo, la diversidad de la creación compartida.

Los miembros de esta corriente se dispersan por una gran variedad de actividades que van desde el artesanado tradicional hasta la electrónica high-tech. Todos ellos utilizan herramientas digitales, diseño onscreen $y$, de forma creciente, la computación para fabricación de máquinas de sobremesa; pero, sobre todo, comparten una norma cultural: la idea de formar parte de una comunidad online que busca mejorar su vida personal y la del entorno mediante la cooperación. Un maker -suelen decir- es creativo, comunicativo (sin secretos), cooperativo y produce bienes sociales.

Quienes forman esta corriente cultural propugnan un aprendizaje abierto, sin jerarquías, y crean comunidades inclusivas a las que puede pertenecer cualquiera; cuentan con medios de difusión (Makezine e Instructables); se reúnen en Makerspaces (centros comunitarios con herramientas y donde se imparten talleres, como el Artisan's Asylum) y organizan eventos (Make Haven) para compartir productos, así como Fablabs para la experimentación. Con ello, asistimos a una democratización de la tecnología y de los instrumentos de manufacturación.

\section{Net neutrality, gobernanza de internet y nueva esfera pública}

No hay duda de que internet ha supuesto una novedad cualitativa en numerosos aspectos de la vida: cualquiera puede editar; ha potenciado y facilitado extraordinariamente la experimentación y la invención colectivas; es multimedia, funciona con hipertexto y puede acumular todas las formas culturales; en cierto sentido, es un bien público puesto que nadie puede controlar su totalidad. Pero ¿la circulación por la red es neutra?, ¿es abierta en sus contenidos e ideología inmanente?, ¿̇los derechos humanos que han funcionado en el mundo físico se garantizan también en ella?, ¿permite construir una esfera pública global?, ¿su carácter descentralizado hace que funcione como una República de Usuarios?

La construcción de una esfera pública en la modernidad estuvo sustentada sobre la conquista de las li- bertades de expresión, de reunión y de asociación. Estas libertades se aprendieron y ensayaron, antes de convertirse en derechos legales, en múltiples espacios y formas de sociabilidad, que recibieron en muchos casos el nombre de "sociedad" porque en ellas justamente se experimentaba la construcción ciudadana de la sociedad y de la política. Durante el siglo XIX se crearon los partidos políticos, pero a finales del XX y principios del XXI estas organizaciones de representación son fuertemente contestadas por su osificación y su sometimiento a los intereses de la banca y las grandes corporaciones.

En los comienzos de internet, los pioneros también ensayaron sin limitaciones políticas sus pautas de funcionamiento y libertades y ante la novedad de la red identificaron la tecnología (arquitectura abierta) con la ideología de la soberanía, la neutralidad y la libertad. ¿Se trataba de un rasgo perdurable, intrínseco, connatural a la propia red o por el contrario de una característica instituyente, asociada a la fase inicial de todo proceso revolucionario?, cera internet una realidad nueva imposible de controlar por los gobiernos? Esta es la pregunta que ha venido haciendo Tim $\mathrm{Wu}$, desde hace tiempo (2010). De hecho, a medida que internet ha ido madurando y se ha convertido en la infraestructura y ecosistema de comunicación y cultura para cualquier aspecto de la vida, han entrado en escena otros actores como los gobiernos, los ejércitos y las grandes multinacionales que ejercen e imponen diversas formas de control sobre la red. "Irónicamente, las que fueron asumidas en tiempos como leyes inmutables de un entorno tecnológico poderoso son ahora especies potencialmente frágiles en un ecosistema amenazado" (Deibert, Palfrey, Rohozinski y Zittrain. 2010, p. 8).

La OpenNet Initiative lleva más de una década estudiando y analizando en profundidad la evolución de internet en función de la regulación del acceso. Además de la etapa inicial del pionerismo (open commons), se habrían producido otras cuatro etapas que han ido alejando la red de las promesas iniciales. EI periodo que va de 2000 a 2005 se caracterizaría por la sistemática creación de filtros defensivos en China, Arabia Saudí, Paquistán y muchos otros países para evitar determinados contenidos (acceso denegado); la siguiente etapa, de 2005 a 2010 (acceso controlado), se define por una estrategia ofensiva de ataques, espionaje, malware, difusión de ideas favorables a los intereses estratégicos de los estados o de las empresas y por el surgimiento de grupos civiles de carácter ultranacionalista que hacen la guerra sucia por su 
cuenta; finalmente, desde 2010 hasta la actualidad, se habría iniciado una nueva etapa (del acceso contestado) en la que se estarían produciendo simultáneamente reacciones de una sociedad civil más madura, de las grandes empresas que se alinean del lado de los derechos civiles (Google, etc.) y de otros gobiernos (Wu, 2010). Un resultado de ello ha sido la creación de la Global Network Initiative, centrada en la defensa de la libertad de expresión y de la privacidad.

La visión de una red distribuida y descentralizada se halla enfrentada, pues, a la realidad de una red crecientemente jerarquizada y centralizada o "feudal" (Bruce Schneier On The Feudal Internet And How To Fight It). En el campo aparecen con fuerza nuevos problemas como el acceso libre, la privacidad (en la medida en que se almacenan datos de nuestras vidas), la seguridad (frente a la omnipresente vigilancia y la ciberdelincuencia), el papel de los algoritmos automáticos, etc. La propia gobernanza de internet es el objeto de una amplia confrontación.

En los países democráticos, los activistas de la cultura libre se oponen a las leyes que promueven la creciente mercantilización y la utilización de la red como instrumento de vigilancia y control; en aquellos que tienen regímenes más o menos represivos, donde se censura y corta el acceso a las redes, donde se persigue y encarcela a los activistas, estos luchan por la pura y simple libertad de acceso. Para todos ellos, la red es el espacio de una nueva ciudadanía -los netizens-, de nuevas formas de movilización y compromiso -el clictivismo-, y de nuevas identidades cívicas -los netactivistas-. Todos ellos defienden que internet es un recurso global universal y debe mantener una arquitectura abierta y que los derechos que las personas tienen en la vida ordinaria también deben ser protegidos online.

La convergencia de muchas organizaciones en las movilizaciones desarrolladas en 2012 en Estados Unidos y en Europa para impedir la aprobación de las ya citadas leyes PIPA, SOPA y ACT, se ha tomado como un momento revelador del nacimiento de una esfera pública digital (networked). El 18 de enero de 2012 Wikipedia, Wordpress y otros sitios apagaron sus páginas durante 24 horas en protesta por las tentativas de control a la libertad de expresión; Google y Fundación Mozilla colocaron bien visibles mensajes de protesta.

Con independencia de la importancia que para el futuro de la internet pueda tener esta batalla puntual, lo cierto es que las nuevas tecnologías permiten la creación de comunidades cívicas y su coordinación para movilizaciones de gran alcance con fines políticos: posibilitan el intercambio de información relevante, proporcionan conectividad social, construyen perspectivas críticas, aseguran la transparencia y rendimiento de cuentas y refuerzan la agencia cívica (Jenkins, 2006). El poder en el mundo digital, al igual que en el mundo físico -sostiene MacKinnon (2012)- debe encontrar el contrapeso de la ciudadanía. Los acuerdos de NETmundial en Brasil ofrecen cierta esperanza sobre el mantenimiento de los principios fundacionales, aunque se ha eludido el término "neutralidad" del documento final; los proyectos de la Federal Communications Commission (FCC) de revisar la neutralidad de la red, por el contrario, constituyen la expresión más patente de las amenazas (Wu, 2010).

\section{LAS APORTACIONES DEL MOVIMIENTO}

En esta síntesis nos centramos en dos de las aportaciones fundamentales del movimiento: una metodológica, que podemos denominar la producción cooperativa de bienes simbólicos; otra referida a la naturaleza del conocimiento, entendido como bien común.

\section{La producción cooperativa de bienes simbólicos}

El movimiento open ha desarrollado, desde sus orígenes, una metodología característica de trabajo. Al tratar de ella, Shirky habla de "organización sin organización", O’Reilly de "arquitectura de participación", Yochai Benkler de Commons-Based-Peer-Production o CBPP, Tapscott y Williams de peering, Reagle de "colaboración de buena fe" y Henry Jenkins de "inteligencia cooperativa". Con estos y otros términos se designa la colaboración generosa de desarrolladores que no pertenecen a una organización empresarial ni institucional, sino que forman parte de redes cuyos límites son porosos y borrosos, cuyos nodos se hallan dispersos en ubicaciones distantes, pero que contribuyen voluntaria y gratuitamente a la producción de un bien común de acceso libre. En este sentido, cada red o comunidad, a diferencia de una organización tradicional, no tiene trabajadores, sino cooperantes.

Estas "comunidades difusas" comparten un principio -apertura- y un modo de ver y de hacer, la conocida como cultura hacker, pero no reúnen las características de las organizaciones burocráticas de mercado, con la correspondiente asignación jerárquica de responsabilidades claras y relaciones definidas y con objetivos únicos. De ahí que pueda dar la impresión de que carecen de estructura. Sin embargo, se basan en una nueva ecología del conocimiento y su estructura se sustenta sobre la competencia o experticia, 
sobre la performance, la experimentación y la revisión constante, y sobre la evaluación y el reconocimiento por pares. Sus productos, como el software o como un puzzle (véase el logo de Wikipedia), son modulares o granulares (componencialidad y ensamblaje), ya que se pueden descomponer en pequeñas tareas que luego se articulan, agregan y rearticulan en proyectos más amplios.

La función de integración de las aportaciones particulares y la de desarrollo de las tareas nucleares fundamentales suele ser obra de un grupo reducido y estable de personas (board), pero la organización se basa en la pertenencia voluntaria, que es fluida y contingente en relación con las tareas ejecutadas, a una red mucho más extensa y de límites borrosos.

Estas comunidades virtuales defienden la apertura como una vía para fomentar la innovación e incentivar las aportaciones; según Auray desarrollan tres lógicas de gobernanza para evitar el vandalismo, los errores comunes y la manipulación estratégica: el control de calidad a posteriori de las versiones estables, que está parcialmente automatizado; la garantía de estatus como reconocimiento simbólico siempre que es posible identificar las aportaciones individuales; y la preferencia por la vigilancia participativa, basada en las aportaciones de base, y buscando el quórum o el consenso (Auray, 2009). Lo que cuenta son las realizaciones, que han de ser visibles y transparentes para someterse al escrutinio y la evaluación de los pares.

Estas redes se basan en estructuras horizontales (de ahí la denominación de comunidades); en la motivación intrínseca y un ethos inclusivo y abierto (los free riders son bien acogidos y se desecha el miedo al fracaso); en un dinamismo de actualización constante del contenido (priman la innovación y la renovación incesantes); en la creación de materiales basados en la cooperación y en la contribución de muchos; en la reutilización de logros y procesos que se hallan siempre disponibles; en la constante adaptación de soluciones digitales para el funcionamiento de la comunidad. Esto ha llevado a algunos autores a hablar de un tercer modo de producción que maximiza el valor de la creatividad humana sin utilizar indicadores como los precios o la gestión burocrática (Benkler 2006; Shirky, 2011). Por su parte, Moulier prefiere integrarlo en la lógica "polinizadora" del capitalismo cognitivo (Moulier Boutang, 2007; Moulier Boutang, 2010).

Un ejemplo eminente de los resultados que pueden producir estos procesos de cooperación se halla en la exitosa enciclopedia Wikipedia. La bibliografía que trata de evaluar su confiabilidad como fuente de información es muy extensa, tanto a favor como en contra (Auray, 2009), pero nadie pone en cuestión su relevancia sociocultural. Parte de una visión: que cada ser humano puede contribuir libremente a la suma total del conocimiento ("cualquiera puede ser editor"); se funda en la tecnología wiki que permite compartir (editar) a gran escala, sin control previo; existen tipos muy distintos de contribuyentes o colaboradores tanto por el nivel de implicación en la edición e introducción de términos como por las funciones que se realizan, pero quienes comparten las normas se conciben a sí mismos como la comunidad de los wikipedianos. ¿Qué normas fundamentales se han dado? Tres: neutralidad de puntos de vista, eludir la originalidad (para ello están las revistas científicas o artísticas) y verificabilidad. Como sostiene Reagle, en cierto sentido "un artículo de Wikipedia no puede ser mejor que sus fuentes" (Reagle, 2010, p. 12); la ambición prometeica de una enciclopedia universal, en este caso, no parece obnubilar el juicio sobre los límites de su realización.

\section{La naturaleza del conocimiento}

"Cada día se nos muestra como mucho más razonable la consideración de que existen cosas, productos y estados de vida económica que comportan o exigen la comunidad" (Altamira, 1890, p. 17).

En la cita introductoria de Alan Greenspan hemos mostrado la creciente importancia que las ideas tienen para la economía contemporánea. En realidad, lo sorprendente, como sostiene Paul M. Romer es el carácter residual que estas han jugado en la teoría económica. Sin embargo, ahora, cuando casi todo puede ser codificado en una serie binaria y el principal recurso es el conocimiento aplicado a la producción de más, nuevo y mejor conocimiento, la cuestión de la propiedad de las ideas se convierte en extraordinariamente relevante.

El conocimiento es tratado, de hecho, con frecuencia como un bien de mercado. Pero, como han mostrado autores tan diversos como Paul Samuelson y Joseph Stiglitz, Paul M. Romer o Richard Florida, se diferencia sustancialmente de otros bienes y objetos. Para ellos, las características diferenciales de las ideas respecto a cualquier otro bien (incluido el capital humano) se derivan de la intangibilidad y se resumen en la no rivalidad y en la imposibilidad de la exclusión. La primera característica conlleva que el consumo por parte de un individuo no impide el de otras personas; la ausencia de exclusividad implica que resulta difícil 
si no imposible apartar a un individuo cualquiera de disfrutar del bien en cuestión.

Las ideas pueden ser usadas o compartidas por mucha gente al mismo tiempo y al utilizarlas no se producen costes marginales, por lo que resulta más eficiente distribuirlas libremente a cualquiera que restringir su uso mediante la imposición de cargas. Cuanto más y mejor es conocida una idea, mejor podrá ser evaluada, mejorada y transformada. Por tanto, la accesibilidad al conocimiento es una garantía de su mejora y de la exploración de las potencialidades de innovación. Por otra parte, la propiedad intelectual más fundamental es la creatividad humana (Florida, 2006).

El movimiento open sostiene que las nuevas tecnologías, al digitalizar objetos y fenómenos, por su instantaneidad y conectividad, explicitan de modo extraordinario ambas características. Sin embargo, las reglas de uso y distribución, en definitiva, las normas sociopolíticas sobre la propiedad intelectual obstruyen el desarrollo de las potencialidades puestas en juego por la revolución digital. La economía actual de la divulgación y de la comunicación científica, por ejemplo, dificulta el acceso y establece filtros selectivos con la consecuencia paradójica de que mayor saber objetivo conlleva menor acceso relativo de los investigadores concretos. Esta paradoja es un resultado de la contradicción entre las potencialidades que generan las tecnologías para una distribución instantánea y el sistema de gestión de la comunicación científica y las reglas reguladoras de la distribución. Viejas reglas y leyes, extendidas y readaptadas, constriñen el desarrollo de relaciones sociales nuevas.

Dos gramáticas operan en el movimiento al abordar estas cuestiones: la del dominio público y la de la comunalidad. No son divergentes, pero es importante señalar algún matiz sobre ellas. Los defensores del dominio público -y muy especialmente Boyle- plantean la necesidad de restringir los derechos de propiedad privada y de ampliar la entrada de las creaciones de la mente (libros, música, películas, etc.) en el dominio público para disfrute de todos; en cambio, entre los partidarios de los commons, se supone que los bienes simbólicos son algo así como los recursos de la naturaleza (dados) y que la comunalidad es la forma originaria de su producción y uso (Altamira, 1890). De hecho, se habla de los comunales digitales por extensión metafórica y se plantea la existencia de un segundo cercamiento por analogía con el que experimentaron bosques y campos al principio de la revolución industrial. En 1990 publicaba Elinor Ostrom su célebre Governing the Commons: The Evolution of Institutions for
Collective Action y en 2001 Lawrence Lessig inventaba las Creative Commons 4 .

Solo si el conocimiento constituye un bien público y una utilidad social y se reconoce el carácter cooperativo de su producción, solo entonces los miembros de dicha sociedad gozarán de los derechos plenos de una ciudadanía que incorpora la condición de internauta.

\section{ORGANIZACIÓN Y BASES SOCIALES DEL MOVIMIENTO}

En un artículo sobre la historia del movimiento (The new politics of the internet. Everything is connected), el articulista de The Economist sostiene que "en la actualidad cada rincón del universo digital tiene su propio grupo de interés". Esto es ciertamente lo que hemos mostrado, pero también que existe una cultura subyacente, más o menos explícita en todas las corrientes y que en ellas opera el principio de apertura, para referirse al conocimiento como bien común, a las formas de producirlo y distribuirlo, y por supuesto a las modalidades de organización cívica y social.

Este principio se extiende desde los grupos y redes que, en un extremo, pueden definirse esencialmente como comunidades de práctica, dado que reúnen a quienes comparten afinidades electivas ("almas gemelas") y encuentran una oportunidad para ejercer sus aficiones, hasta, en el otro extremo, las redes cívicas y los hackers anónimos que defienden causas y proyectos de transformación social, cuyos beneficios se postulan para el conjunto de la sociedad (Ariño Villarroya, 2009).

A su vez, es posible diferenciar una corriente más o menos libertaria que postula la gratuidad total de todos los bienes simbólicos y que conecta con una creciente sensibilidad social reticente al pago, y una corriente más liberal, que distingue entre libertad y gratuidad. Esta última no excluye la recompensa económica al autor, sino que plantea la instauración de límites o la reformulación del derecho de propiedad intelectual y una revisión drástica del papel lucrativo desempeñado por las entidades de gestión de derechos.

Esta complejidad interna, funcional e ideológica, refleja las condiciones de emergencia de internet. Castells ha señalado la existencia de cuatro capas culturales: la universitaria tecnomeritocrática (la ciencia por amor a la ciencia), la de los hackers (es decir, la de quienes tienen la pasión de crear), la de las formas culturales alternativas (comunidades virtuales) capaces de inventar nuevas formas sociales y la empresarial orientada a hacer negocio mediante la innovación. Estas cuatro capas, aunque son distintas, se 
van reforzando y comparten la cultura de la libertad, para la cual internet debe ser una tecnología abierta, sin apropiación privada (Castells, 2008). A ellas, como hemos visto, habría que añadir una quinta, la de los gobiernos, centrada por ahora más en la vigilancia y el control que en los principios de apertura y comunalidad, con los consiguientes conflictos.

En el seno de este conglomerado de organizaciones y de vínculos más o menos fuertes o débiles, se puede distinguir un centro y muchas periferias; cada rama, corriente o proyecto tiene límites porosos por razones estratégicas: incorporar la novedad. De acuerdo con las teorías clásicas de las organizaciones, a medida que crecen en volumen y en complejidad, también se debería incrementar la jerarquía y la burocracia. Sin embargo, esto ya no sucede con "las organizaciones sin organización" (Shirky, 2008) porque las nuevas aplicaciones permiten coordinar la acción de forma instantánea, a distancia, sin necesidad de estructuras burocráticas, tanto de personas intensamente participativas como de otras que realizan aportaciones casuales e incluso parásitas y oportunistas.

En el interior del movimiento hallamos individuos carismáticos, geniales y extraordinariamente creativos, con frecuencia extravagantes -véase la fiesta de Burning Man $^{5}$-, que ejercen un liderazgo reconocido, como redes de trabajo y cooperación, de afinidad en gustos o prácticas, que utilizan el código abierto para comunicarse y operar; se da tanto la existencia de amplias bases de voluntariado, anónimas, dispuestas a efectuar pequeñas aportaciones y donaciones a un proyecto conjunto y grupos estables, con capacidad ejecutiva y centralizados; también hallamos fundaciones, universidades y organismos estatales. Las bibliotecas universitarias asociadas en redes de diverso tipo juegan un papel importante en el ámbito de la apertura de la comunicación científica. En este sentido, puede hablarse de un movimiento de estructura rizomática, que combina dimensión carismática e institucional; individual y organizacional; local y global. Y todo ello lo hace merced a las potencialidades de las nuevas tecnologías, que son a un tiempo su condición de operación y su meta, su infraestructura técnica, su nicho ecológico y su contenido.

Sin embargo, en estas redes sí que siguen operando las leyes clásicas de distribución del poder (Pareto). Estas leyes -sostiene Shirky- tienden a funcionar en todos los sistemas sociales donde muchas personas expresan sus preferencias entre muchas opciones; cuando el número de estas crece, la curva de distribución se hace más extrema. Si aumenta el volumen del sistema también crece el gap entre el $1 \%$ y la media (Shirky, 2008). Para explicar esta desigual participación, Fuster ha acuñado el concepto de ecosistema participativo en el sentido de subrayar la codependencia y la adaptación mutua de diferentes formas y grados de contribución, en orden a lograr un equilibrio entre ellas para alcanzar la sostenibilidad y la efectividad de la misión común.

En un cierto sentido, no es posible ignorar que muchos de los líderes del movimiento forman parte de organizaciones que les procuran libertad y autonomía (departamentos e institutos universitarios) y otros se han dotado de estructuras organizativas que les permiten operar fuera del campo estrictamente mercantil, como es el caso de las fundaciones. Además de que la mayoría son varones, suelen compartir dos rasgos más: han nacido en la década de los cincuenta (y se han visto influidos por el ethos contracultural de los campus norteamericanos de finales de los sesenta y primeros setenta); y todos ellos tienen formación universitaria y en algún momento han desarrollado o desarrollan tareas de formación e investigación en centros universitarios.

Por ello mismo, el movimiento adopta formas de desarrollo que han probado su indudable éxito en el mundo profesional y académico: funciona mediante encuentros, foros, seminarios y congresos, donde los pares presentan los resultados de sus procesos de trabajo y debaten abiertamente sobre ellos; se crean asociaciones profesionales; se redactan declaraciones y manifiestos, a los que se adhieren quienes están acordes con su filosofía siguiendo pautas típicamente universitarias; se crean revistas y repositorios; etc. Pero también se utilizan las potencialidades de las nuevas tecnologías para la difusión cotidiana y para la comunicación ordinaria: blogs, weblogs, wikis, foros, listas, aplicaciones, comunidades virtuales, etc. Al mismo tiempo, se actúa dentro de las instituciones u organizaciones de las que se forma parte -universidades y otras organizaciones públicas- ejerciendo influencia y presión para que estas adopten políticas explícitas de apoyo al movimiento. En cierto sentido, podría decirse que han llevado al extremo la lógica de la democratización en la evaluación académica por pares, puesto que no son ahora los expertos solamente quienes tienen la última palabra sino cada participante.

Esta práctica a ultranza de la apertura organizativa no deja de crear problemas, como muestra el caso de Wikipedia, generados por quienes utilizan las puertas abiertas para quebrar la cultura de la cooperación y la presunción de bondad en toda acción humana. A 
ello hacen frente con nuevas estrategias organizativas y nuevas aplicaciones técnicas, pero también surgen organizaciones donde existen filtros de control (WikiLeaks) o el anonimato.

Finalmente, algunos sectores están tratando de dar el salto a la esfera política creando partidos específicos. Este es el caso de los partidos piratas en algunos países nórdicos y Alemania o Austria entre otros.

Nos hallamos, pues, ante un movimiento que no suele utilizar las formas típicas de acción colectiva no convencional -manifestaciones, ocupaciones, protestas, etc.- sino que se sirve con eficacia de los resortes que proporciona la red y de las estrategias profesionales de sus miembros, recurriendo en ocasiones a pautas típicas de grupos de presión. Esto no es extraño en absoluto, y no solo por la pertenencia institucional de muchos de sus líderes, sino porque como sostiene Suber, el movimiento no es revolucionario en sus demandas, aunque posiblemente lo sea en sus consecuencias.

\section{CONCLUSIÓN}

El movimiento open, en el conjunto de sus ramificaciones y corrientes, aborda el impacto de las tecnologías que crean una cultura global digital sobre las formas de producción y difusión del conocimiento y sobre las formas de organización social.

En el primer caso, se ocupa de cómo se transforman los procesos de distribución (copias sin coste marginal, cambios en los soportes, quiebra en la cadena de distribución, nuevos modelos de negocio) y los procesos de producción: cómo se genera el conocimiento (metodologías cooperativas, autoría colectiva, lógica del $20 / 80$ ) y qué se produce (nuevos objetos de conocimiento como el genoma, la física subatómica, los exoplanetas, el medio ambiente, etc.) y, en consecuencia, qué es el conocimiento y cuál es su naturaleza, quién es el autor de los bienes simbólicos y cuáles son sus derechos, y quién produce conocimiento (modificación de la relación entre producción y consumo).

En el segundo, se plantean las cuestiones del acceso a la red, de la seguridad y la privacidad, pero sobre todo de la compatibilidad entre los principios de la democracia y de los derechos humanos y la creciente regulación centralizada y jerárquica de la internet. Por ello se plantean cómo crear una esfera pública global, como incrementar el dominio público, cómo favorecer la autonomía personal y la ciudadanía.

El movimiento open, desde los pioneros del open commons hasta las movilizaciones en numerosos paí- ses en 2013-2014 contra el creciente control de la red, desde las comunidades de fans, pasando por las de software, y llegando a las distintas formas de protesta digital, reclama la importancia de una internet pública y libre, donde haya autores, creadores y artistas que ponen sus conocimientos y creaciones al servicio de la comunidad de internautas; donde la dinámica cooperativa, las comunidades virtuales, la producción CBPP, etc. adquieran un papel determinante; donde el conocimiento generado con fondos públicos sea accesible y gratuito; en definitiva, una internet donde el bien común del conocimiento y de la innovación derivada, desde la nueva esfera pública global, permita crear una sociedad mejor.

Existe, pues, un movimiento global centrado en el principio de apertura, que se ramifica abordando las distintas problemáticas que surgen en la red y sobre la red, pero el carácter excepcional de esta, concebida como la única estructura descentralizada, distribuida, que no puede ser sometida al control de las grandes multinacionales para discriminar la circulación de contenidos en función de los precios (no de los costes), que es soberana frente a los estados, cuando vemos cómo estos desarrollan distintas estrategias de vigilancia generalizada sin garantías jurídicas de ningún tipo, ya no puede ser asumido como un hecho, sino como un "ideal que debe ser defendido" (Wu, 2010, p. 187).

No es objetivo de este artículo evaluar el alcance del movimiento open. No obstante, no es posible concluir sin señalar que internet hoy en día está presente real o potencialmente en todo el mundo y es una infraestructura global de la cultura, pero todavía no es coextensiva con la sociedad mundial; y tampoco se puede ignorar que una parte importante de la información y la comunicación tiene objetivos socialmente perversos, manipulada por las profundas fuerzas y poderes que han dominado en la historia humana; o que la gran mayoría de ella, en clave de transformación social, es banal e irrelevante; que el malware y la desinformación estratégica también ocupan sus nodos. Y, en fin, como sostienen Gasser, Faris y Heacock, que "en la mayoría de cuestiones y lugares, la esfera pública networked permanece dormida" (Gasser, Faris y Heacock, 2013, p. 9).

\section{AGRADECIMIENTOS}

Este artículo se enmarca en la investigación del proyecto NOMS (Nuevos Objetos Mundo-Sociales), financiado por el Ministerio de Economía y Competitividad. 


\section{NOTAS}

1 Términos frecuentes son los de Software Free, Free Culture, Creative Commons, Science Commons, Information Commons, Public Knowledge, Libre Knowedge y Public Domain

2 La visión inspiradora de la declaración para el conocimiento libre reza como sigue: "knowledge for all, freedom to learn, towards collective wisdom, enabling communities to empower themselves with knowledge" (Declaration on libre knowledge).

3 Un mapa mundial de repositorios, revistas y recursos Open Access en http://

\section{BIBLIOGRAFÍA}

Altamira, R. (1890). Historia de la propiedad comunal. Madrid: Arte y ciencia.

Ariño Villarroya, A. (2009). El movimiento Open. La creación de un dominio público en la era digital. Valencia: Publicacions Universitat de València.

Anderson, C. (2012). Makers: The New Industrial Revolution. New York: Crown Business.

Atkins, D. E., Brown, J. S. y Hammond, A L. (2007). A review of the Open Educational Resources (OER) Movement: Achievements, Challenges, and New Opportunities [En línea]. Disponible en https://hewlett.org/wp-content/ uploads/2016/08/ReviewoftheOERMovement.pdf

Auray, N. (2009). Le Web participatif et le tournant néo-libéral : des communautés aux solidarités. En Proulx, S., Millerand, F. y Rueff, J. (dirs.), Web relationnel : mutation de la communication ? Presses Universitaires de Québec, pp. 11-48. Disponible en http://ses.telecom-paristech.fr/auray/2009AurayWebParticipatif.pdf

Benkler, Y. (2006). The Wealth of the Networks. How Social Production Transforms Markets and Freedom. Yale University Press.

Camaerts, B. y Meng, B. (2011). Creative destruction and copyright protection: regulatory responses to file-sharing. London: LSE.

Castells, M. (2008). The New Public Sphere: Global Civil Society, Communication Networks, and Global Governance. The ANNALS of the American Academy of Political and Social Sci- www.openaccessmap.org/; http:// oanow.org/; http://www.openaccess. nl/; http://scoap3.org/; http://www. hefce.ac.uk/news/newsarchive/2014/ news86805.html; https://royalsociety. org/news/2014/royal-society-openscience/

4 Entre otras publicaciones sobre el tema véase Mattei (2011), Dardot y Laval (2014), la tesis de Mayo Fuster en http://www.onlinecreation.info/ outline design y numerosas páginas web: http://thinkcommons.org/; http://onthecommons.org/. En España

ence, 616 (1), pp. 78-93. https://doi. org/10.1177/0002716207311877

Dardot, P. y Laval, C. (2014). Commun. Essai sur la révolution au XXlème siècle. $\mathrm{Pa}$ ris : La Découverte.

Deibert, R., Palfrey, J., Rohozinski, R. y Zittrain, J. (2010). Access Controled, The Shaping of Power, Rights, and Rule in Cyberspace. Cambridge, Massachusetts: The MIT Press. https://doi. org/10.7551/mitpress/8551.001.0001

Fernández, M. (2012). Sistemas de bajo coste: Fab Lab Valencia. [Tesis de maestría inédita]. Universidad Politécnica de Valencia: Valencia.

Florida, R. (2006). The rise of the creative class. Basic Books.

Gershenfeld, N. (2012). How to Make Almost Anything. The Digital Fabrication Revolution. Foreign Affairs, 91 (6), pp. 43-57.

Gasser, U. Faris, R. y Heacock, R. (2013). Internet Monitor 2013: Reflections on the Digital World. The Berkman Center for Internet and Society at Harvard University. Research Publication 2013-27. Disponible en https://papers.ssrn.com/ sol3/papers.cfm?abstract_id $=2366840$

Hess, C. y Ostrom, E. (eds.) (2006). Understanding Knowledge as a Commons. From Theory to Practice. Cambridge, Massachusetts /London, England: The MIT Press. https://doi.org/10.7551/ mitpress/6980.001.0001

Jenkins, H. (2006). Culture Convergence. Where Old and New Media Collide. New York: New York University Press.

Lessig, L. (2005). Por una cultura libre. Madrid: Traficantes de sueños. el procomún https://www.medialabprado.es/programas/laboratorio-delprocomun. Para un reportaje sobre los comunales en Europa, véase http:// communia-project.eu/final-report/.

5 El Burning Man es un festival anual que se desarrolla durante siete días en Black Rock City, en Nevada, Estados Unidos. Black Rock City no es un municipio ni tiene gobierno, solo existe durante la semana de Burning Man en medio del desierto de Nevada, a 150 kilómetros al noreste de Reno. La edición de 2015 congregó a más de 70.000 personas.

MacKinnon, R. (2012), No sin nuestro consentimiento. La lucha por la libertad en internet. Bilbao: Deusto.

Mattei, U. (2011). Beni comuni. Un manifesto. Roma: Laterza.

Moglen, E. (2003). Freeing the Mind: Free Software and the Death of Propietary Culture. Fourth Annual Technology and Law Conference, Portland, Maine, 29 de junio. [En línea]. Disponible en http:// moglen.law.columbia.edu/publications/maine-speech.html

Moulier Boutang, Y. (2007). Le capitalisme cognitif. La nouvelle grande transformation. Paris: Amsterdam.

Moulier Boutang, Y. (2012). La abeja y el economista. Madrid: Traficantes de Sueños.

Ostrom, E. (1990). Governing the Commons: The Evolution of Institutions for Collective Action. Cambridge University Press. https://doi.org/10.1017/ CB09780511807763

Raymond, E. S. (2001). The Cathedral and the Bazaar. Musings on Linux and Open Source by an Accidental Revolutionary. New York: O’Reilly.

Romer, P. M. (1992). Two strategies for Economic Development: Using Ideas and Producing Ideas. The World Bank Economic Review, 6 (suppl. 1), pp. 6391. https://doi.org/10.1093/wber/6. suppl_1.63

Shirky, C. (2008). Here comes everybody. The power of organizing without organizations. London: Penguin.

Shirky, C. (2011). Cognitive Surplus. Creativity and Generosity in a Connected Age. London: Penguin. 
Suber, P. (2012). Open Access. Boston: The MIT Press. https://doi.org/10.7551/ mitpress/9286.001.0001

Wiley, D. A. (2000). Connecting learning objects to instructional design theory: A definition, a metaphor, and a taxonomy. The Instructional Use of Learning $\mathrm{Ob}$ jects, 2830 (435), pp. 1-35.

Wu, T. (2010). Is Internet Exceptionalism Dead? En Szoka, B. y Marcus, A. (eds.), The Next Digital Decade. Essays on the future of the Internet. TechFreedom, pp. 179-188.

\section{Otros recursos}

Blanc, S. (2011, 11 de marzo). [App] Quand I'Internet se manifeste [v1]. Owni. [En línea]. Disponible en: http://owni. $\mathrm{fr} / 2011 / 03 / 11 / a p p$-quand-linternet-se-manifeste-v1/

Bruce Schneier On the Feudal Internet and How to Fight It. Techdirt. [En línea]. Disponible en: https://www.techdirt. com/articles/20131025/08383625015/ bruce-schneier-feudal-internet-how-tofight-it.shtml
COAPI principles. [En línea]. Disponible en http://www.sparc.arl.org/sites/default/ files/COAPIPrinciples\%20\%281\%29.pdf

Declaration on libre knowledge. [En línea]. Disponible en http://www.wikieducator org/Declaration_on_libre_knowledge

Directory of Open Access Journals (DOAJ). [En línea]. Disponible en: https://doaj.org/

Global Multistakeholder Meeting on the Future of Internet Governance. Netmundial. [En línea]. Disponible en http://netmundial.br/es/

Nicol, A., Caruso, J. y Archambault, E. (2013). Open Data Access Policies and Strategies in the European Research Area and Beyond. August 2013. European Comission DG Research \& Innovation RTD-B6PP-2011-2: Study to develop a set of indicators to measure open access. [En línea]. Disponible en: http://www.science-metrix.com/pdf/SM EC OA Data.pdf

Suber, P. Open Access Overview. Focusing on open access to peer-reviewed research articles and their preprints. [En línea]. Disponible en: https://goo.gl/io2zob
Subirats i Coll, I. El movimiento Open Access: Concepto e historia. Septiembre 2007. [En línea]. Disponible en: http:// sabus.usal.es/bib_virtual/doc/subirats_ open.pdf

The Federal Reserve Board (2004). Intellectual property rights. Remarks by Chairman Alan Greenspan at the Stanford Institute for Economic Policy Research Economic Summit, Stanford, California. February 27, 2004. [En línea]. Disponible en: http://www. federalreserve.gov/Boarddocs/Speeches/2004/200402272/default.htm

The new politics of the internet. Everything is connected. The Economist, 5 enero 2013. [En línea]. Disponible en: http:// www.economist.com/node/21569041/ print

Wheeler, T. (2014, 24 de abril). Setting the Record Straight on the FCC's Open Internet Rules. Federal Communications Commission. [En línea]. Disponible en https://www.fcc.gov/news-events/ blog/2014/04/24/setting-recordstraight-fccs-open-internet-rules 